\title{
Use of an automatic device for the determination of mechanical properties on photoelastic samples of small dimensions
}

\author{
Felipe Maia Prado*1@, Daniel José Toffoli ${ }^{1}$, Sidney Leal da Silva ${ }^{1}$ \\ ${ }^{1}$ Fatec Itaquera, Grupo de óptica e aplicações, São Paulo, SP, Brasil.
}

Received on March 10, 2020. Revised on July 14, 2020. Accepted on August 18, 2020.

\begin{abstract}
Photoelastic materials and Photoelasticity have been very effective in the study of the effects of stress and strain in materials such as iron, steel, concrete, etc. However, mainly because of human interaction, systematic errors are introduced during the measurement process, which directly affects the final result. When using small photoelastic samples, these types of errors can escalate due to the increased difficulty of handling. Intending to minimize systematic errors in the process of data collection, this work presents the use of an automatic loading device, developed by us, to apply mechanical stresses in small photoelastic samples. The device created has shown great effectiveness in photoelastic applications that uses a transmission polariscope, but it can also be used in other photoelastic techniques, such as reflection photoelasticity, as well as in other areas of optics such as Holography and Speckle. An important benefit is the ease and speed of handling that can improve the organization and quality of the activities in labs of optics.
\end{abstract}

Keywords: Photoelasticity, Photoelastic materials, Mechanical elasticity, Polarization.

\section{Introduction}

Photoelastic materials and Photoelasticity are alternatives in the study of material properties such as elasticity modulus and Poisson's coefficient ${ }^{1,2}$. Photoelastic materials are widely used in industry for indirect determination of properties of materials such as iron, steel, concrete etc. $^{3,4}$, or in the dentistry for the study of maxillary stresses distributions due to chewing forces ${ }^{5,6}$.

This huge interest in photoelasticity comes from the transparency and high flexibility the photoelastic materials show, which allows the observation of the phenomenon of temporary double refraction by polarization, or birefringence, first described by Erasmus Batholinus in the 19 th $^{\text {century }}{ }^{7,8}$ and associated with the state of stress and deformation of the material by David Brewster ${ }^{9}$. Photoelasticity is a subarea of optics that studies the internal effects caused by external forces on samples of photoelastic materials using polarized light ${ }^{10,11}$.

However, it was only in the 1950s that Photoelasticity assumed an important role in science and technology ${ }^{12,13,14}$. In the $1970 \mathrm{~s}$, digital processing was incorporated into Photoelasticity, which allowed a better interpretation of the results. In the $1980 \mathrm{~s}$, optical instruments, called polariscopes, composed of combinations of polarizers and wave plates were incorporated into photoelasticity, making it modern and closer to how it is known today ${ }^{15}$.

*Correspondence email address: felipemp369@gmail.com
The use of conventional photoelasticity techniques requires excessive human interaction, causing systematic

errors in data collection. Another difficulty presented by these techniques is evidenced by the inadequacy for samples with small dimensions, in common load devices.

This paper presents an automatic loading device to assist in the collection of data for small photoelastic samples, under the action of external forces, reducing human interaction and, consequently, minimizing systematic errors, contributing to more reliable final results. This loading device, created by us, was used in a transmission polariscope, in which green light (filtered from a white light source) incides into the small photoelastic sample, a rectangular sheet of epoxy resin. By the use of errors theory ${ }^{16,17}$ the collected data were analyzed using a computer program and the modulus of elasticity and Poisson's coefficient were determined. This loading device can also be used in other subareas of Optics, such as Holography and Speckle.

\section{Theory}

Birefringence is the phenomenon of double refraction by polarization, intrinsic in certain crystalline anisotropic materials, such as calcite $\left(\mathrm{CaCO}_{3}\right)$, and temporary in isotropic materials under stress, such as epoxy resins. In these materials, the incident radiation is divided into two wavefronts, with polarization states perpendicular to each other ${ }^{10,11}$. 
Figure 1 shows a cut of calcite revealing the effect of intrinsic birefringence.

Figure 2 shows a parallelepiped of epoxy resin with and without external stress showing the effect of temporary birefringence.

In 1816 researcher Sir David Brewster showed that generally transparent isotropic materials can become anisotropic, when strained, and thus display characteristics of birefringent materials ${ }^{9}$. When light goes through these materials, colored fringes (isochromatic) and black ones (isoclinic) can be observed with the aid of polarizers. These fringes present dynamic behavior and shift as the stresses are applied in the material, as shown in Figure 2. The branch of optics that relates the observed fringes to the stresses and deformations that occur within temporarily birefringent materials, also called photoelastic materials, is Photoelasticity ${ }^{10,11}$.

Optical information obtained by Photoelasticity is related to the differences between the internal stresses considered in the longitudinal, $\sigma_{l}$, and transversal, $\sigma_{t}$, directions, by the Optical Stress Law ${ }^{19}$,

$$
n_{l}-n_{t}=C\left(\sigma_{l}-\sigma_{t}\right)
$$

$\mathrm{C}=\mathrm{C}(\lambda)$ is the photoelastic dispersion coefficient, which is dependent on wavelength, $\lambda$ of the light; $\left(n_{l}-n_{t}\right)$ is the difference between refractive indices considered in the longitudinal, $n_{l}$, and transversal, $n_{t}$, directions.

In photoelasticity, the refractive index values are not obtained directly and thus existing methods produce results of stress differences by indirect processes ${ }^{15}$.

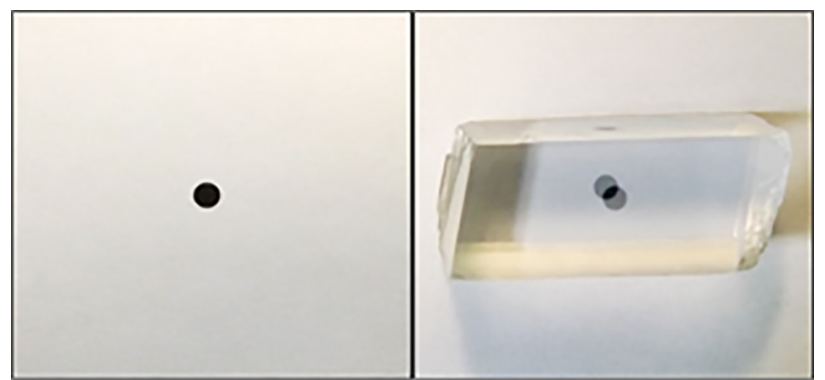

Figure 1: Dot on a white sheet of paper (left); the effect of birefringence caused by the calcite (right). (photos from reference ${ }^{18}$, authorized by the author)

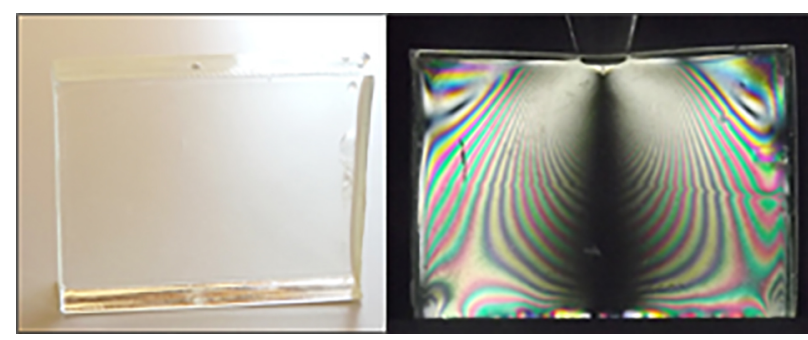

Figure 2: Parallelepiped of epoxy resin without any load (left); and under external tensions (right). (photos from reference ${ }^{18}$, authorized by the author)
One alternative to determining the optical properties of the photoelastic materials, presented in this paper, involves capturing information directly from the images of the static fringes associated with each external force applied at the photoelastic sample, and store in digital format. These forces generate longitudinal and transverse stresses within the material, by the theory of the plane state of $\operatorname{stresses}^{20}$. For data processing, the model used is shown in Figure 3.

By Robert Hooke's law ${ }^{2}$

$$
\sigma=E \cdot \varepsilon
$$

where $\mathrm{E}$ is the modulus of elasticity of the material and $\varepsilon$ is the linear deformation in the longitudinal direction. $\varepsilon$ is related to the original length of the linear material, such that

$$
\varepsilon=\frac{\ell-\ell(0)}{\ell(0)}=\frac{\Delta \ell}{\ell(0)}
$$

where $\Delta \ell$ represents the variation in length $\ell$ and $\ell(0)$ represents the original length of the material. As there is deformation in the transversal direction to the application of the force, by the theory of the plane state of $\operatorname{stresses}^{20}$, the relationship between the longitudinal and transversal deformation is a constant, in the elastic regime, such that

$$
\varepsilon_{\text {longitudinal }}=-\nu \cdot \varepsilon_{\text {transversal }}=-\nu \cdot \frac{\sigma}{E},
$$

where $\nu$ is the Poisson's coefficient of the material. The sign (-) adjusts the expression, since generally positive stresses produce negative deformations, and vice versa.

To determine the modulus of elasticity and the Poisson's coefficient, the external stresses applied to the photoelastic sample and the respective deformations, both longitudinal and transverse, were used. The mean deformations can be calculated from values obtained by lines of pixels along a chosen direction of the static fringes image, outlined in Figure 4, caused by the stresses applied to the photoelastic sample.

Each pixel line, shown in Figure 4, is associated with a relative intensity, $I_{R}$, according to the diagram in Figure 5 .

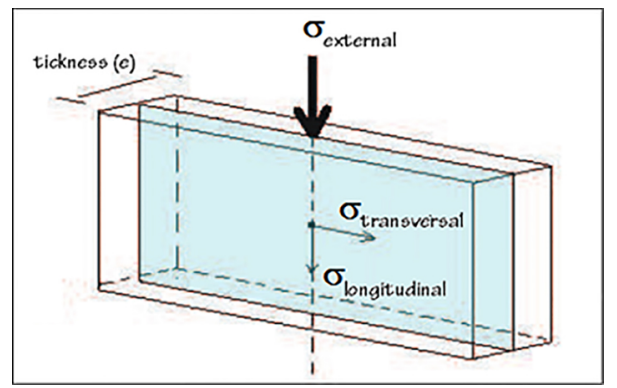

Figure 3: Theoretical model for a photoelastic sample. (scheme modified from the reference ${ }^{18}$, authorized by the author) 


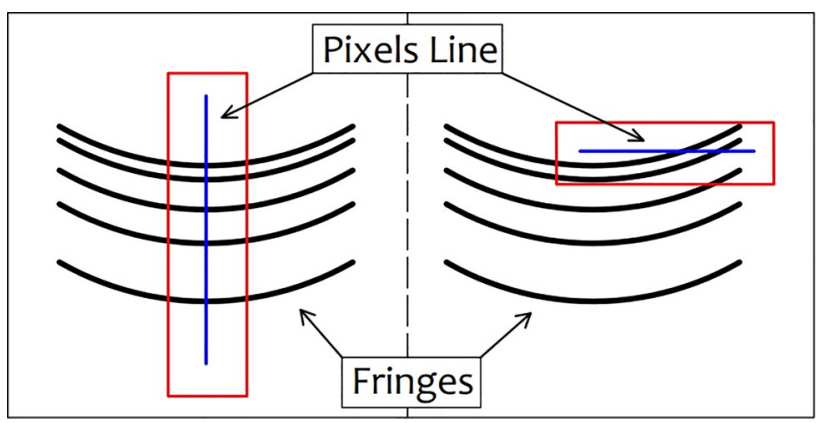

Figure 4: Line of pixels to determine the longitudinal mean deformation (left side diagram); line of pixels to determine the transversal mean deformation (right side diagram)

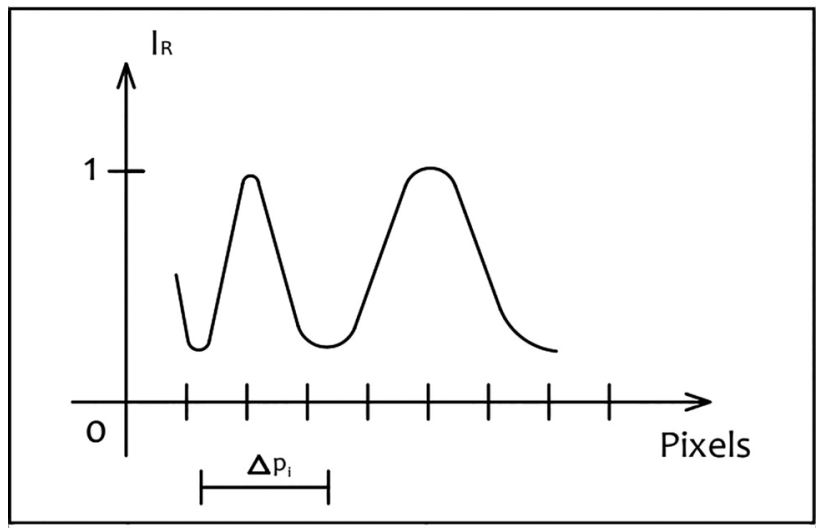

Figure 5: Representation of the $I_{R}$ curve versus ordered pixels

The i-th relative intensity is determined from the equation,

$$
I_{R_{i}}=\frac{I_{\max }-I_{i}}{I_{\max }}
$$

where $I_{i}$ represents the i-th intensity, for the i-th pixel, with $\mathrm{i}=1,2, \ldots \mathrm{n}$. $I_{\max }$ represents the maximum intensity of the selected pixel line.

As illustrated in Figure 5, taking the pixels values of consecutive valleys, or peaks, of intensity and subtracting them, we obtain the difference between the pixel positions, $\left.<\Delta p_{t}\right\rangle$, for a specific fringe created by a tension $\mathrm{t}$. The application of each load is repeated a-number of times, therefore, the mean width, $\left\langle\Delta d_{t}\right\rangle$, of a fringe for one specific value of tension is determined by the equation,

$$
<\Delta d_{t}>=\frac{1}{n} \sum_{a=1}^{n} \Delta p_{t_{a}}
$$

To determine the mean strain, $\langle\varepsilon>$, of each image, the following equation is used,

$$
<\varepsilon>=\frac{<\Delta d_{t}>}{d_{\text {reference }}},
$$

where $d_{\text {reference }}$ is the value of $\left\langle\Delta d_{t}\right\rangle$ for the highest load applied in the sample. Equation (7) is an approximation that can be derived assuming that the deformation field of a bar, placed horizontally and supported at its two ends, under tension is similar to the fringe pattern produced when we apply tension to a photoelastic sample. Thus, just as the deformation of the bar can be calculated by the difference in lengths, equation (3), the deformations in the photoelastic material can be calculated in terms of the differences of pixels values. This approximation is derived in detail in the work of Da Silva (2017).

To determine the elasticity modulus, $E$, of the photoelastic sample, Hooke's Law represented in Equation (2) is used, such that

$$
<\sigma_{j}>=E \cdot\left\langle\varepsilon>_{j}\right.
$$

where $\langle\sigma\rangle_{j}$ is the $\mathrm{j}$-th external mean stress and $\langle\varepsilon\rangle_{j}$ is the $j$-th mean strain, on the photoelastic sample, with $\mathrm{j}=1,2, \ldots \mathrm{m}$. From Equation (8), a graph similar to the schema in Figure 6 can be obtained.

By Equation (8) and chart scheme of Figure 6,

$$
\mathrm{E}=\operatorname{tg}(\gamma)
$$

To determine the Poisson's coefficient, $\nu$, of the photoelastic sample, the module of the Equation (4) is used, such that

$$
<\varepsilon_{\text {longitudinal }}>_{j}=\nu \cdot<\varepsilon_{\text {transversal }}>_{j}
$$

where $<\varepsilon_{\text {longitudinal }}>_{j}$ is the $\mathrm{j}$-th mean longitudinal strain and $<\varepsilon_{\text {transversal }}>_{j}$ is the $\mathrm{j}$-th mean transversal strain. From Equation (10), a graph similar to the schema in Figure 7 can be obtained.

By Equation (10) and chart scheme of Figure 7,

$$
\nu=\operatorname{tg}(\gamma)
$$

In both graphs, the best fits and $\operatorname{tg}(\gamma)$ were obtained by the Least Square Method ${ }^{16,17}$.

\section{Methodology}

A rectangular sheet of epoxy resin, produced by a polymerization process, was used as a photoelastic sample. This resin offers excellent adhesion to a large number of

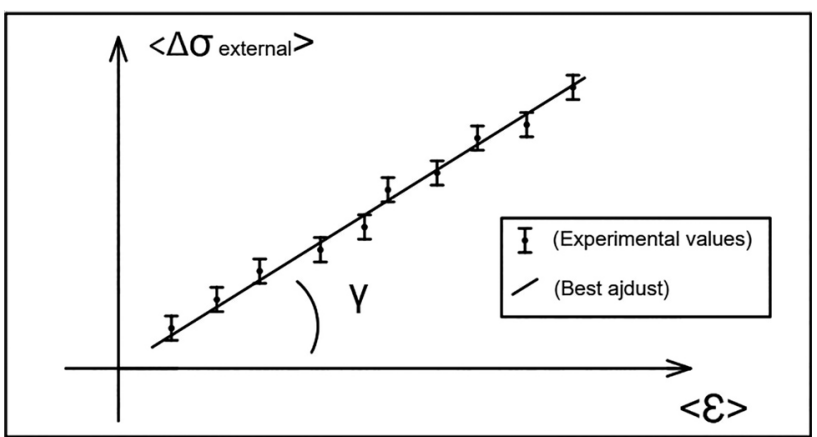

Figure 6: Graph with experimental values and best fit by minimum squares method ${ }^{16,17}$ to $\sigma_{\text {external versus } \varepsilon}$ 
materials and exhibits optical fringes when subjected to external stresses. Details on the making of the photoelastic sample are described in Da Silva et $a .^{21}$.

Figure 8 presents the photograph of the photoelastic sample used for the experiment.

The automatic loading device in Figure 9 has two different ways of applying stress to small photoelastic samples: sequential and continuous. In sequential mode, the stepper motor applies predetermined stress values on the photoelastic sample.

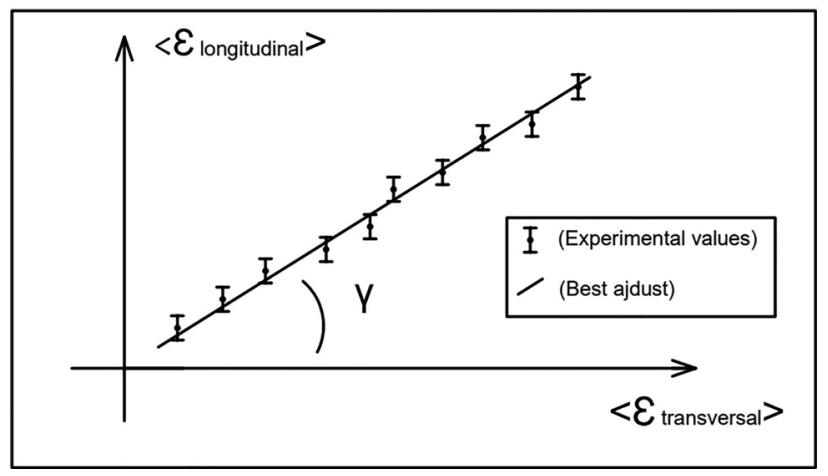

Figure 7: Graph with experimental values and best fit by minimum squares method ${ }^{16,17}$ to $\varepsilon_{\text {longitudinal }}$ versus $\varepsilon_{\text {transversal }}$

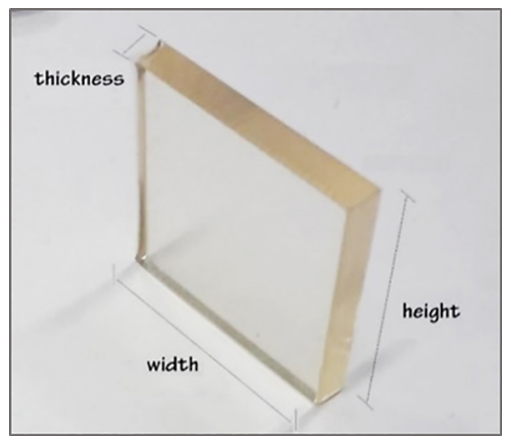

Figure 8: photoelastic sample of dimensions $0.485 \times 3.176 \times$ $3.014\left(10^{-2} \mathrm{~m}\right)$ (thickness $\times$ width $\times$ height)

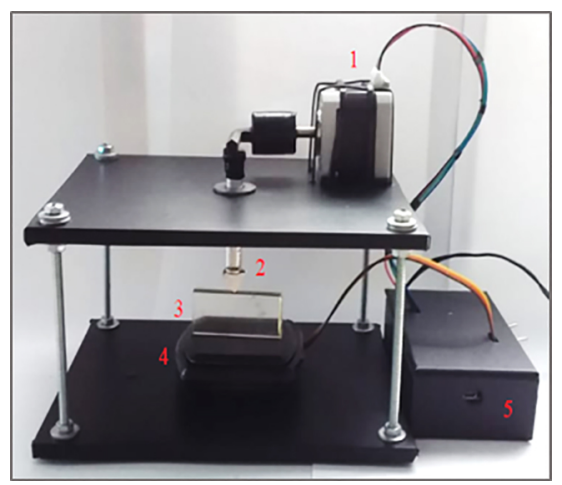

Figure 9: load device to perform the compressions of the sample (1 - stepper motor Nema 17; 2 - compression tip; 3 - photoelastic sample; 4 - load cell; 5 - electronic circuit)
With each new load addition, the mechanism is stopped for a certain period of time so that the photographic capture of the image can be performed. In continuous mode, the loads exerted on the sample vary constantly and uniformly, with increments in the order of tenths of a second, and the images are captured in video form.

Figure 10 shows a photograph of the polariscope configuration used to collect data with the automatic loading device attached to the configuration.

The wavefront, produced by a white light source, (1), is altered by the neutral density filter and lens, (2), becoming approximately flat at the sample due to the small thickness, if compared with its height and width. Then, a color filter, (3), allows the passage of light, within a certain range of wavelengths, so that only fringes of the same color are observed. Then, two linear polarizers, (4) and (6), with orthogonal polarization states prevent, a priori, the passage of light.

Under the force provided by the device, the polarization state of the light changes as it passes through the sample (A), allowing the digital camera to observe the isochromatic fringes produced. With the addition of two quarter-wave plates, (5), before and after the sample, the optical configuration becomes that of a circular polariscope and, therefore, the isoclinic fringes are eliminated $^{22}$. Allowing a purer image of the isochromatic fringes. The images are captured by the digital camera and stored in digital files for future analysis by the computer system.

The proposed method used computational analysis associated with error theory ${ }^{16,17}$ treatment. In this work the continuous mode of the automatic device was selected, so the images were stored in video digital files. A number of frames, Q, out of the total frames, P, produced by the video were chosen from the consecutive compressions on the photoelastic sample. G groups, each containing Q/G image frames, were separated for the data processing.

The following topics explain how the mechanical elasticity modulus and the Poisson's coefficient were determined.

a) Determination of the mechanical elasticity modulus

For each group G of images, the computer system:

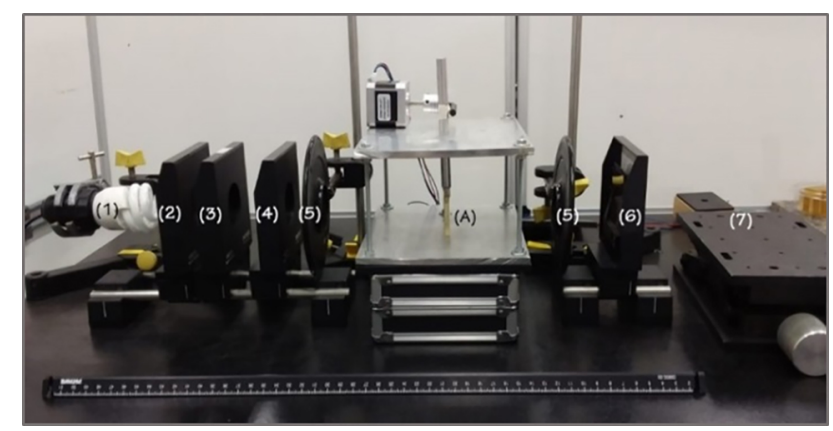

Figure 10: The load device for small photoelastic samples in the polariscope by transmission configuration 


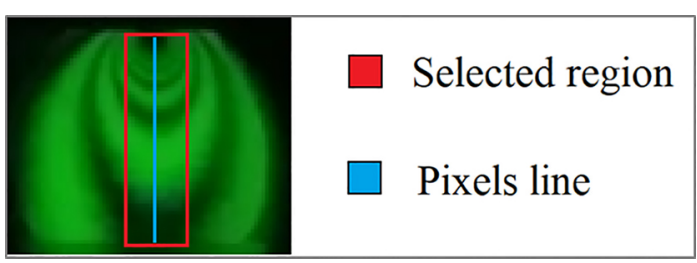

Figure 11: Photo of the fringes with the rectangular selection and line of pixels chosen in a frame

1. Selected the region for study, the same in all images, as shown in Figure 11. Selecting the line right in the center of the sample ensures that $\sigma_{\text {longitudinal }}$ » $\sigma_{\text {transversal }}$, according to the model shown in Figure 3.

2. The selected images were converted to the gray pattern with $\mathrm{N}$ bits, $\mathrm{M}$ shades of gray;

3. With the line of selected pixels, according to the scheme of Figure 11, graphs of relative intensity, $I_{R}$, versus ordered pixels were constructed, as shown in the scheme of Figure 5. The $I_{R}$ values were calculated by Equation (5), where the $\mathrm{I}_{\mathrm{Max}}$ was the maximum pixel intensity in the selected line;

4. In each $\mathrm{I}_{\mathrm{R}} \mathrm{x}$ pixels graph, distances $\Delta p_{t a}$ were determined and, with this, the mean distances $<$ $\Delta d_{t}>$ were calculated by Equation (6), as well as their respective uncertainty by the error theory ${ }^{16,17}$ process.

5. From the computation of the $\mathrm{Q} / \mathrm{G}$ frames of each group, the mean longitudinal strains, $\langle\varepsilon\rangle$, were determined by Equation (7), with their respective uncertainties.

6. The mean external stresses, in each frame group, were determined by the mean relation between the force modules, carried out by the loading device, and the area of operation of the compression pointer. Each frame is associated with a mean compression force determined by the stepper motor of the loading device;

7. The relationship between the mean external stresses versus mean longitudinal strains was represented in a graph of experimental values, properly transferred to the dependent parameter, in this case the mean stress, according to the scheme of Figure 6;

8. A linear regression generated the best fit for the experimental points and, with that, the elasticity module of the photoelastic sample was obtained, with its respective uncertainty.

\section{b) Determination of the Poisson's coefficient}

For each group $\mathrm{G}$ of images, the computer system:

1. Selected the region for study, the same in all images, as shown in Figure 12. Selecting a region near the top of the sample ensures $\sigma_{\text {transversal }}$ $» \sigma_{\text {longitudinal }}$, according to the model shown in Figure 3.

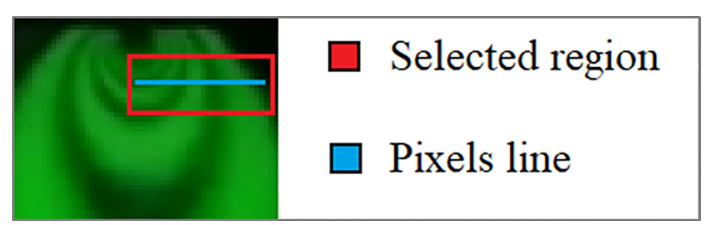

Figure 12: Photo of the fringes with the rectangular selection and line of pixels chosen in a frame

2. To determine the mean transversal strains, procedures like those presented in sub-topics (ii) to (v) of item a) were performed;

3. The relationship between the mean longitudinal strains (calculated in item a) versus mean transversal strains were represented in a graph of experimental values with the uncertainties, properly transferred to the dependent parameter, in this case the mean longitudinal strain, according to the scheme of Figure 7;

4. A linear regression generated the best fit for the experimental points and, with that, the Poisson's coefficient of the photoelastic sample was obtained.

\section{Results}

A digital caliper, with an uncertainty of $0.01 \mathrm{~mm}$, was used to determine the width, height, and thickness of the photoelastic sample used in this work. To justify the statistical process of gaussian distribution, used to obtain the final results, through error theory ${ }^{16,17}$, all dimensions were measured 10 times. Table 1 shows the obtained dimensions.

The video obtained by the digital camera produced $\mathrm{P}=4096$ frames, of which were selected $\mathrm{Q}=4080$, divided into $\mathrm{G}=12$ groups of $\mathrm{Q} / \mathrm{G}=340$ frames. The images were converted to the standard containing 256 gray shades, 8 bits.

The graphs in Figure 13 show the relationships between the external mean stresses versus longitudinal mean strains and transverse mean strains versus longitudinal mean strains, respectively, for the sample in Figure 8.

One can notice that in both graphs the experimental values are distributed around the adjusted line, showing that the system is credible and, therefore, with good quality of adjustment, by the method of least squares. Adjustments were made with the functions $\mathrm{f}(\mathrm{x})=\mathrm{ax}$ and $\mathrm{f}(\mathrm{x})=\mathrm{ax}+\mathrm{b}$. The results proved to be more reliable with the function $\mathrm{f}(\mathrm{x})=\mathrm{ax}$, due to the proximity to the

Table 1: Geometric parameters of the test photoelastic sample

\begin{tabular}{lc}
\hline & $\mathbf{1 0}^{-\mathbf{2}} \mathbf{~ m}$ \\
\hline width & $3.176 \pm 0.009$ \\
height & $3.014 \pm 0.007$ \\
thickness & $(4.85 \pm 0.06) 10^{-1}$ \\
\hline
\end{tabular}



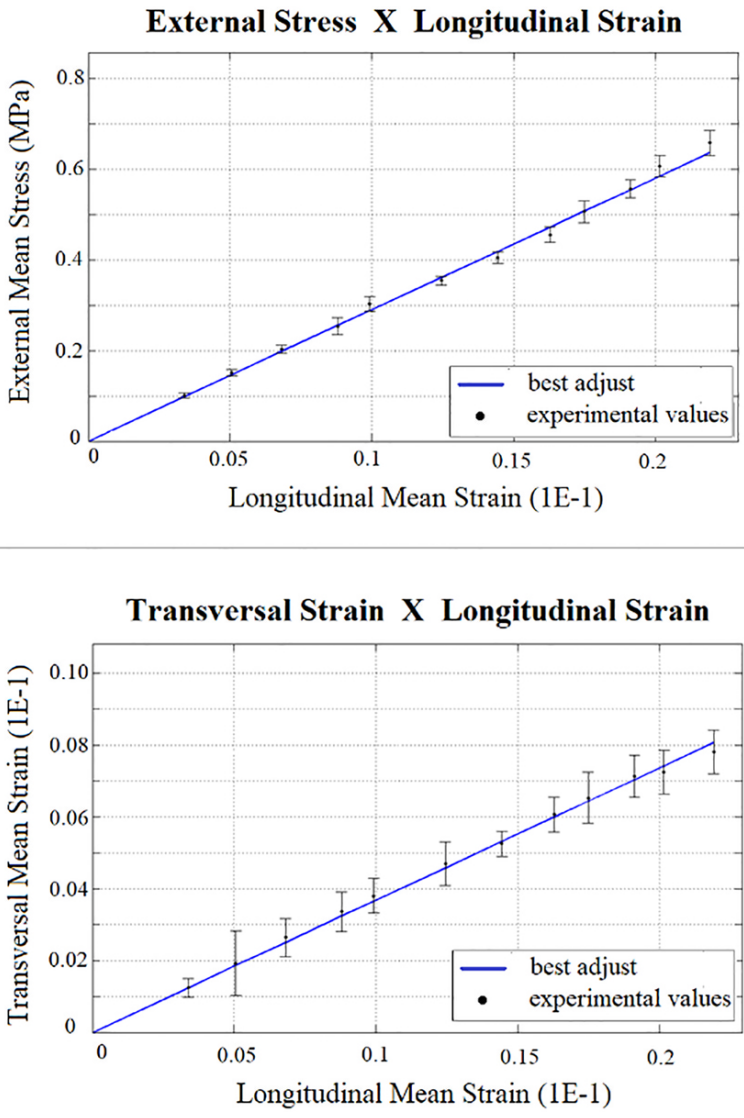

Figure 13: Graphs of external mean stresses versus longitudinal mean strains and transversal mean strain versus longitudinal mean strain for the sample in Figure 8

Table 2: Modulus of elasticity and Poisson's coefficient

\begin{tabular}{lc}
\hline$<\mathbf{E}>(\mathbf{M P a})$ & $29.02 \pm 0.34$ \\
$<\boldsymbol{\nu}>\left(\mathbf{1 0}^{-\mathbf{1}}\right)$ & $3.68 \pm 0.11$ \\
\hline
\end{tabular}

Table 3: Modulus of elasticity and Poisson's coeficiente from the work of Da Silva (2017)

\begin{tabular}{lcc}
\hline & I & II \\
\hline$<\mathbf{E}>(\mathbf{M P a})$ & $27.9 \pm 0.7$ & $33.1 \pm 0.1$ \\
$\langle\boldsymbol{\nu}\rangle\left(\mathbf{1 0}^{-\mathbf{1}}\right)$ & $3.876 \pm 0.068$ & $3.891 \pm 0.073$ \\
\hline
\end{tabular}

generalized Hooke's law, $\sigma_{\text {extern }}=E \cdot \varepsilon_{\text {transversal }}$, by Thomas Young ${ }^{2,20}$, and, $\varepsilon_{\text {longitudinal }}=\nu \cdot \varepsilon_{\text {transversal }}$, by Siméon Poisson ${ }^{2,20}$.

The results obtained for the mean modulus of elasticity, $\langle\mathrm{E}\rangle$, and mean Poisson's coefficient, $\langle\nu\rangle$, according to equations (9) and (11), are shown in Table 2.

Table 3 presents the values of Modulus of elasticity and Poisson's coefficient obtained in the literature ${ }^{18}$ for a photoelastic sample, similar to the one used in this work.

Although the values, between this work and those found in the literature, have been generated by different methods and techniques, they present the same order of magnitude. With different coefficients and uncertainties, as expected. Comparing the values of Table 2 with the ones shown in Table 3, the relative differences between the modulus of elasticity are, approximately, $3.92 \%$ for the value I and $12.46 \%$ for the value II. For the Poisson's coefficients, the relative differences are, approximately, $5.14 \%$ and $5.51 \%$ for the values I and II, respectively.

\section{Conclusions and Perspectives}

The values obtained agree, in order of magnitude, with results found in the literature for photoelastic samples, even for different dimensions and concentrations of the sample used. The automatic loading device fitted very well at the application, that involved the photoelastic configuration, as can be observed in Figure 10 and proved to be stable during the measurement process, facilitating the work of efficient data collection. A small sample adapts easily to the device, which allows for easy removal and placement, resulting in time savings in data collection.

The greater amount of data generated by the automatic device, about 40 times greater than the collection with the usual mechanical devices made in our previous works, produced results with greater accuracy. The lesser human interaction decreased some systematic errors during measurements and reduced processing times during analysis.

The presented method demonstrated great efficiency in the quality and reliability of the results, allowing values comparable to those of the literature, thanks to the computational system produced.

A possible improvement in the device can be obtained by more rigorous machining to facilitate producing controls of movements through computational means. Another possible improvement in the method can be carried out by creating a data control system through a relational database, which will allow a better organization of the processing, allowing faster production of the final result. Other optical techniques, such as Photoelasticity by reflection, Speckle by transmission and reflection, and Holography by transmission and reflection, can be tested with the device produced.

\section{Acknowledgments}

Special thanks to senior researcher Dr. Eduardo Acedo Barbosa, professor at Fatec São Paulo, who encouraged and supported the work.

\section{References}

[1] S.S. Pawar, N.V. Patil and H.V. Shete, International Research Journal of Engineering and Technology 4, 1475 (2017).

[2] W.D. Callister, Fundamentos da ciência e engenharia de materiais: uma abordagem integrada (LTC, Rio de Janeiro, 2012), $2^{\mathrm{a}}$ ed. 
[3] H.A.F. Toro, J.C.B. de León, A.R.R. Martínez and J.W.B. Bedoya, Visión electrónica 11, 89 (2017).

[4] N. Parthasarathi, S.S. Senthil, M. Prakash and K.S. Satyanarayanan, Asian Journal of Civil Engineering 19, 647 (2018).

[5] J. Lee, Y. Lee, Y.L. Kim and H.W. Cho, The Journal of prosthetic dentistry 115, 161 (2016).

[6] G.V. Portes, E.M. Boeck, N. Lunardi and K.E.D.C. Pizzol, Revista Brasileira Multidisciplinar 20, 113 (2017).

[7] B.A. Moura, Revista Brasileira de Ensino de Física 36, 1 (2014).

[8] F. Bréhat and B. Wyncke, Représentation des états de polarisation des ondes lumineuses (Publibook, Paris, 2003), $1^{\mathrm{a}}$ ed.

[9] D. Brewster, Philosophical Transactions of the Royal Society of London 105, 1 (1815).

[10] B.E.A. Saleh and M.C. Teich, Fundamentals of Photonics (Wiley \& Sons, New Jersey, 2007), $2^{\text {a }}$ ed.

[11] E. Hecht, Óptica (Fundação Calouste Gulbenkian, Lisboa, 2002), $2^{\mathrm{a}}$ ed.

[12] M.M. Froncht and R.J. Fuernsey, NACA Annual Report 39, 963 (1953).

[13] E.G. Coker and L.N. Filon, A treatise on Photo-Elasticity (University Press, Cambrige, 1957), $2^{\text {a }}$ ed.

[14] L. Pöppl and E. Mönch, Praktische Spannungsoptik (Springer, Heldelberg, 1972), $1^{\mathrm{a}}$ ed.

[15] K. Ramesh, T. Kasimayan and B.N. Simon, The Journal of Strain Analysis for Engineering Design 46, 245 (2011).

[16] J.H. Vuolo, Fundamentos da teoria de erros (Blucher, São Paulo, 2003), $2^{\text {a }}$ ed.

[17] F.J. Macwilliams and N.J.A. Sloane, The theory of errorcorrecting codes (Elsevier, Amsterdã, 1977), $1^{\text {a }}$ ed.

[18] S.L. Da Silva, Estudo de tensões em amostras fotoelásticas com Holografia Digital (Novas Edições Acadêmicas, São Paulo, 2017), $1^{\mathrm{a}}$ ed.

[19] A. Kuske and G.S. Robertson, Photoelastic stress analysis (Wiley-Interscience, Hoboken, 1974), $1^{\text {a }}$ ed.

[20] H.L. Soriano, Método de Elementos Finitos em Análise de Estruturas (Edusp, São Paulo, 2003), 1a ed.

[21] S.L. Da Silva et. al., Revista Brasileira de Física Tecnológica Aplicada 2, 1 (2015).

[22] J.W. Dally and W.F. Riley, Experimental stress analysis (Mc-Graw-Hill, Singapore, 1991), $3^{\text {a }}$ ed. 